\title{
Boundary string current \& Weyl anomaly in six-dimensional conformal field theory
}

\author{
Chong-Sun $\mathrm{Chu}^{a, b}$ and Rong-Xin Miao ${ }^{c}$ \\ ${ }^{a}$ Physics Division, National Center for Theoretical Sciences, \\ National Tsing-Hua University, Hsinchu 30013, Taiwan \\ ${ }^{b}$ Department of Physics, National Tsing-Hua University, \\ Hsinchu 30013, Taiwan \\ ${ }^{c}$ School of Physics and Astronomy, Sun Yat-Sen University, \\ Zhuhai, 519082, China \\ E-mail: cschu@phys.nthu.edu.tw, miaorx@mail.sysu.edu.cn
}

ABSTRACT: It was recently discovered that for a boundary system in the presence of a background magnetic field, the quantum fluctuation of the vacuum would create a nonuniform magnetization density for the vacuum and a magnetization current is induced in the vacuum [1]. It was also shown that this "magnetic Casimir effect" of the vacuum is closely related to another quantum effect of the vacuum, the Weyl anomaly. Furthermore, the phenomena can be understood in terms of the holography of the boundary system [2]. In this paper, we generalize this four dimensional effect to six dimensions. We use the AdS/BCFT holography to show that in the presence of a 3-form magnetic field strength $H$, a string current is induced in a six dimensional boundary conformal field theory. This allows us to determine the gauge field contribution to the Weyl anomaly in six dimensional conformal field theory in a $H$-flux background. For the $(2,0)$ superconformal field theory of $N$ M5-branes, the current has a magnitude proportional to $N^{3}$ for large $N$. This suggests that the degree of freedoms scales as $N^{3}$ in the $(2,0)$ superconformal theory of $N$ multiple M5-branes. The prediction we have for the Weyl anomaly is a new criteria that the $(2,0)$ theory should satisfy.

Keywords: AdS-CFT Correspondence, Anomalies in Field and String Theories, MTheory

ARXIV EPRINT: 1812.10273 


\section{Contents}

1 Introduction 1

2 Boundary string current 4

3 Holographic boundary current 5

4 Weyl anomaly from boundary current $\quad 8$

$\begin{array}{llr}5 & \text { Discussion } & 9\end{array}$

$\begin{array}{ll}\text { A Derivation of key relation (4.2) } & 11\end{array}$

$\begin{array}{ll}\text { B Holographic Weyl anomaly } & 13\end{array}$

\section{Introduction}

The decoupling limit of $N$ coincident M5-branes is given by an interacting $(2,0)$ superconformal theory in 6 dimensions [3]. For a single M5-brane, the low energy theory is known and is given by a free theory of tensor multiplet [4-9]. The multiple M5-branes theory is much more complicated and it is not expected to have a fundamental in terms of a local Lagrangian description. ${ }^{1}$ There exists a number of proposals for the fundamental formulation of the six dimensional $(2,0)$ theory: most notably, these include the Discrete Light-Cone Quantisation definition based on quantum mechanics on the moduli space of instantons [16, 17], a definition based on deconstruction from four dimensional superconformal, quiver field theories [18], and the conjecture that the $(2,0)$ theory compactified on a circle is equivalent to the five dimensional maximally supersymmetric Yang-Mills theory $[19,20]$. And despite an extensive amount of work on this topic, see for example, [2138], the field theoretic description of the multiple M5-branes system remains mysterious. In addition to consistency and symmetry requirement, the fundamental theory, no matter how it is defined, should reproduce properties that are expected of the multiple M5-branes system. For example, it should describe a non-trivial interacting theory of $(2,0)$ superconformal multiplets. It should contain BPS states of self-dual strings which corresponds to boundaries of M2-branes ending on the stack of M5-branes [39, 40]. It should explain

\footnotetext{
${ }^{1}$ However just like in supergravity or hydrodynamics, it is perfectly sensible to look for a classical Lagrangian description for the effective dynamics of the $(2,0)$ fields in the long wavelength limit. In this context, see for example [10] where a set of non-Abelian self-dual equation has been constructed and proposed as the classical equation of motion for the self-dual tensor gauge field in the low energy effective theory of the multiple M5-branes theory, with various supporting evidences obtained in [10-15]. We remark however that supersymmetry is missing in this construction.
} 
the $S$ duality of the $\mathcal{N}=4$ supersymmetric Yang-Mills theory [41]. It should also make apparent the $N^{3}$ entropy behaviour [42]. In particular it should explain whether this is due to novel degrees of freedom of the $(2,0)$ theory or not. One of the motivations of this work has been to add new criteria to the list by uncovering new physical properties of the multiple M5-branes system.

To this end, we recall an useful approach that has been known to work very well in the past is to introduce a boundary to the system. For example, the form of the noncommutative geometry, including the relation between closed string and open string metric, on D-branes worldvolume can be derived by considering open strings ending on the D-branes [43, 44]. Therefore we are motivated to consider a M5-branes system with boundary. The resulting low energy theory is a boundary conformal field theory (BCFT), which is a very interesting class of theories by itself.

Boundary conformal field theory $[45,47]$ describes the fixed point of renormalization group (RG) flow in boundary quantum field theory and has important applications in quantum field theory, string theory and condensed matter system such as, for example, renormalization group flows and critical phenomena [45] or the topological insulator [46]. For general shape of the boundary, traditional perturbative analysis of BCFT becomes exceedingly complicated. In addition to traditional field theory techniques, see, e.g. [4855], the need of a non-perturbative approach using symmetries or dualities is evident. A non-perturbative holographic dual description to BCFT was initiated by Takayanagi in [56] and later developed for general shape of boundary geometry in $[57,58]$. The duality has been extensively studied in the literature, with many interesting results obtained. See, for example [59-69].

The Casimir effect is one of the most well known manifestation of the quantum fluctuation of vacuum in the presence of boundary [70-72]. Recently the Casimir effects has been analyzed in full generality for arbitrary shape of boundary and for arbitrary spacetime metric. Universal relations between the Casimir coefficients which determine the near boundary behaviour of the renormalized stress tensor and the boundary central charge in a boundary conformal field theory have been discovered [50]. The analysis has also been extended to $\mathrm{U}(1)$ current in BCFT [1]. It was found that when an external magnetic field is applied, the vacuum of BCFT will get magnetized and a magnetization current get induced in the vicinity of the boundary. In analogous to the standard Casimir effect which is a manifestation of the mechanical property of the vacuum, this effect is a manifestation of the magnetic property of the quantum vacuum and may be refereed to as a magnetic Casimir effect. The generalization of this effect to higher dimensions was another motivation of this work.

The above described effects for the stress tensor and the U(1) current can be derived from the AdS/BCFT holography [2, 50]. From the field theory point of view, they can also be derived from the Weyl anomaly of the BCFT [1,50]. Consider a CFT with partition function $Z\left[g_{\mu \nu}\right]$ and the effective action $W\left[g_{\mu \nu}\right]=\ln Z\left[g_{\mu \nu}\right]$. The scaling symmetry of CFT is generally broken due to quantum effects and the breaking is measured by the Weyl anomaly

$$
\mathcal{A}:=\left.\partial_{\varphi} W\left[e^{2 \varphi} g_{\mu \nu}\right]\right|_{\varphi=0}=\int_{M}\left\langle T_{\mu}^{\mu}\right\rangle
$$


The metric contribution to the Weyl anomaly is well understood. For example in even dimensions, the bulk part of the Weyl anomaly takes the form [73]

$$
\left\langle T_{\mu}^{\mu}\right\rangle=\frac{1}{(4 \pi)^{d / 2}}\left(\sum_{j} c_{d j} I_{j}^{(d)}-(-1)^{\frac{d}{2}} a_{d} E_{d}\right) .
$$

Here $E_{d}$ is the Euler density in $d$ dimensions, $I_{j}^{(d)}$ are independent Weyl invariants of weight $-d$ and the subscript $j$ labels the Weyl invariants. The boundary terms of the Weyl anomaly has also been studied and classified recently in [49]. In general, in addition to a nontrivial background metric, one may also turn on a gauge field background and the loops of matter fields will give a Weyl anomaly. For example in 4 dimensions, vector gauge field (Abelian or non-Abelian) is classically conformal and there is a Weyl anomaly [74]

$$
\left\langle T_{\mu}^{\mu}\right\rangle=b \operatorname{tr} F^{2} .
$$

Here $b=\beta(g) / 2 g^{3}$ and $\beta(g)$ is the beta function of the theory $S=-1 /\left(4 g^{2}\right) \int \operatorname{tr} F^{2}$. For higher $d=2 n$ even dimensions, a $n$-form gauge field $H$ is conformal invariant classically. One can expect a background of $H$-flux will give rises to a Weyl anomaly. However since we do not even know what the higher rank gauge field couple to and how, let alone the quantisation, nothing is known about the possible form of this anomaly. The wish to say something about this Weyl anomaly from background of higher form gauge field has been another major motivation of this work.

That this goal can be achieved follows from the observation in [1] that the gauge part of the Weyl anomaly is intimately related with the induced current, see (4.1) for 4-dimensions. Similar anomaly-current relation can be straightforwardly established for higher dimensions. In the case of six dimensions where we are interested in, we can introduce a boundary to the CFT and first use AdS/BCFT to compute the induced string current, and then use this result and the anomaly-current relation to determine the gauge field contribution to the Weyl anomaly in 6-dimensional CFT.

The plan of this paper is as follows. In section 2, we first review the phenomena of induced current in 4 dimensions. We then generalize it to six dimensions. We show that the use of symmetries and conservation law of the theory allows us to fix, up to a few numerical coefficients, the form of the one point function of a conserved current in the presence of a background of 3-form flux. In section 3, we use AdS/BCFT holography to determine the form of the induced current. The result is consistent with the form obtained by the field theory analysis. In section 4, we generalize the relation between Weyl anomaly and the conserved current to six dimensional BCFT. Using this relation and the result of the induced current from AdS/BCFT as input, we obtain the contribution of the 3-form field strength to the Weyl anomaly in six dimensional CFT. For the system of maximal $(2,0)$ supersymmetric multiple $N$ M5-branes, the current and the Weyl anomaly are found to be proportional to $N^{3}{ }^{2}$ This provides some evidence that the fundamental degree of freedoms of the $(2,0)$ theory of $N$ M5-branes scales like $N^{3}$.

\footnotetext{
${ }^{2}$ The $N^{3}$ dependence has also been found for the gravitational contribution to the conformal anomaly in the Coulomb branch of the $(2,0)$ theory by relating the Coulomb branch interactions in six dimensions to interactions in four dimensions using supersymmetry [30, 75-77].
} 


\section{Boundary string current}

Consider a boundary conformal field theory (BCFT) defined on a manifold $M$ with boundary $P$. In [1], we have shown that for 4 -dimensions, the vacuum expectation value of the renormalized current $J_{\mu}$ has the asymptotic expansion near the boundary at $x=0$,

$$
\left\langle J_{\mu}\right\rangle=\frac{\alpha_{1}}{x} F_{\mu \lambda} n^{\lambda}+\cdots,
$$

when a background gauge field strength $F_{\mu \nu}$ is turned on. Here $\cdots$ denotes terms that are less singular. It was shown that the current (2.1) is related to the Weyl anomaly (1.3) and the coefficient $\alpha_{1}$ is completely determined in terms of the beta function of the theory. It was also understood that the current (2.1) is a consequence of the magnetization of the vacuum which arises from the quantum fluctuation of the vacuum in the presence of the boundary. Here we are interested in generalizing this phenomena of near boundary current to higher dimensional BCFT in the presence of a higher form gauge field background.

Let us consider a 6-dimensional BCFT with gauge symmetry defined on a manifold $M$. Yang-Mills gauge field is not conformal invariant in six dimensions, instead a 2 -form gauge field $B_{\mu \nu}$ is. For simplicity, we consider Abelian gauge field here. The 2-form gauge potential is naturally coupled to the worldsheet $\Sigma$ of a string with the minimal coupling

$$
I_{B}=\int_{\Sigma} B=\int_{M} J^{\mu \nu} B_{\mu \nu}
$$

where

$$
J^{\mu \nu}=\lambda \epsilon^{\alpha \beta} \frac{\partial X^{\mu}}{\partial \sigma^{\alpha}} \frac{\partial X^{\nu}}{\partial \sigma^{\beta}} \delta^{(4)}\left(X-X\left(\sigma^{a}\right)\right)
$$

is a two-form string current that arises from the motion of the string and $\lambda$ is the string charge density. Next let us introduce a boundary $P=\partial M$. This breaks the bulk conformal symmetry and the one point function of the current can become nontrivial now. As the current $J_{\mu \nu}$ has a mass dimension 4 , the vacuum expectation value of the renormalized current generally takes the form

$$
\left\langle J_{\mu \nu}\right\rangle=\frac{1}{x} J_{\mu \nu}^{(1)}+\log x J_{\mu \nu}^{(0)}+\cdots
$$

near the boundary. Here we have used gauge invariance and the conservation law

$$
D_{\mu} J^{\mu \nu}=0
$$

to rule out terms like $J_{\mu \nu}^{(4)} / x^{4}, J_{\mu \nu}^{(3)} / x^{3}, J_{\mu \nu}^{(2)} / x^{2}$. In (2.4), $\cdots$ denotes terms that are regular at $x=0$, and $J_{\mu \nu}^{(1)}$ and $J_{\mu \nu}^{(0)}$ are functions of dimension 3 and 4 respectively. Their form are constrained by (2.5) and the Lorentz and gauge symmetries of the theory. For example, one can easily determine that

$$
J_{\mu \nu}^{(1)}=\alpha_{1} H_{\mu \nu \lambda} n^{\lambda}+\alpha_{2} \mathcal{D}_{[\mu} \mathcal{D}_{\nu]} k+\alpha_{3} \mathcal{D}_{[\mu} \mathcal{D}_{\lambda} k^{\lambda}{ }_{\nu]}+\alpha_{4} \mathcal{D}_{\lambda} \mathcal{D}_{[\mu} k^{\lambda}{ }_{\nu]},
$$

where $H_{\mu \nu \lambda}, n_{\mu}, \mathcal{D}_{\mu}, k_{\mu \nu}$ are respectively the background 3 -form field strength, normal vector to the boundary, induced covariant derivative and the extrinsic curvature of the boundary. The coefficients $\alpha_{i}$ are arbitrary and contain important physical information of the 


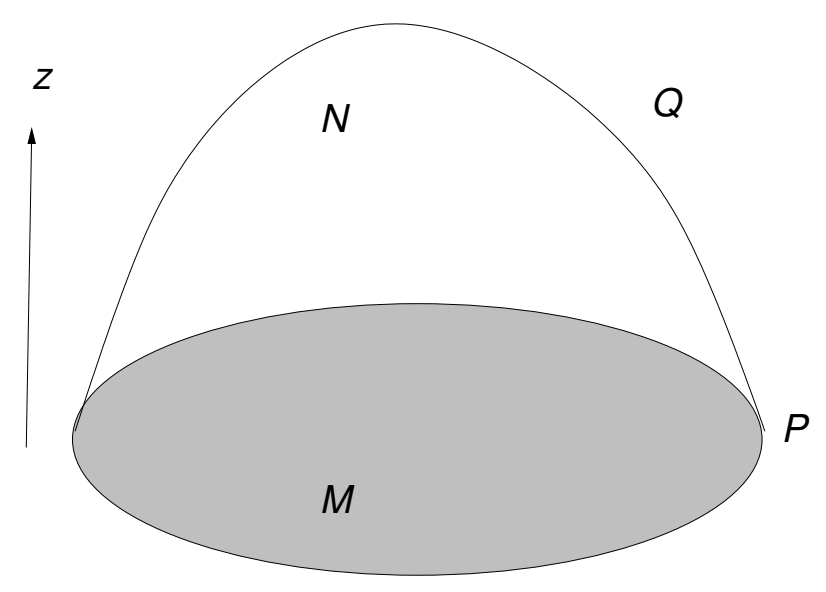

Figure 1. BCFT on $M$ and its dual $N$.

theory. In [1] it was shown that, for four dimensions, the near boundary asymptotic form of the standard current $J_{\mu}$ is completely determined by the background field strength of the Weyl anomaly. It was also shown in [2] that the near boundary current can also be determined using the AdS/BCFT holography. For six dimensions, the background gauge field part of the Weyl anomaly is unknown. Therefore let us proceed first with the holographic analysis and determine the near boundary current using boundary holography.

\section{Holographic boundary current}

Holographic dual of BCFT was originally introduced by Takayanagi [56]. The idea is to extend the $d$ dimensional manifold $M$ to a $(d+1)$-dimensional asymptotically AdS space $N$ such that $\partial N=M \cup Q$, where $Q$ is a $d$ dimensional manifold with boundary $\partial Q=\partial M=P$. According to the standard AdS/CFT arguments, the asymptotic boundary behaviour of a bulk field $\phi$ in AdS generates the expectation value of local operator $\mathcal{Q}$ in the CFT. In our case, let us consider a 2-form tensor gauge field in the bulk described by the gravitational action

$$
I=\frac{1}{16 \pi G_{N}} \int_{N}\left(R-2 \Lambda-\frac{1}{6 q^{2}} \mathcal{H}_{L M N}^{2}\right) .
$$

Here $G_{N}$ is the Newton constant in 7 dimensions, $1 / q^{2}$ is a dimensional constant of length dimension 4 , and $\mathcal{H}=d \mathcal{B}$. $\mathcal{B}$ is the bulk gauge field whose boundary value is given by the gauge field $B$ on the boundary $M$. Note that $B$ is completely arbitrary and does not need to satisfy any equation of motion. The bulk indices are denoted by the capital Roman letters $L, M, N=0,1, \cdots, 6$ and the indices of the 6-dimensional manifolds $M$ and $Q$ are denoted by Greek letters $\mu, \nu$ etc. It should be clear from the context whether we are referring to the manifold $M$ or $Q$. The existence of the tensor gauge field in the bulk dictate the presence of a 2 -form current $J_{\mu \nu}$ in the CFT whose expectation value is determined by the generating relation,

$$
Z_{\text {string }}\left[B_{\mu \nu}\right]=\left\langle e^{\int_{M} J^{\mu \nu} B_{\mu \nu}}\right\rangle .
$$


In field theory, the current is constructed as the Noether current of some global Abelian symmetry. In this paper, we will be interested in the SUGRA limit where the string partition function is given by the SUGRA action (3.1).

The new ingredient in AdS/BCFT is that the SUGRA action isn't defined until the shape $Q$ is known. According to Takayanagai [56], $Q$ is determined as an extremal configuration of the supergravity action with respect to change of $Q$ :

$$
16 \pi G_{N} I=\int_{N}\left(R-2 \Lambda-\frac{1}{6 q^{2}} \mathcal{H}_{L M N}^{2}\right)+2 \int_{M} K+2 \int_{Q}(K-T)+2 \int_{P} \theta .
$$

Here the constant parameter $T$ is a measure of the boundary degree of freedom of the BCFT.

To specific the variational principle, one needs to fix the boundary condition for $Q$. As $N$ is of codimension one, the location of $Q$ is determined by a single function. A consistent model of holographic BCFT was found by considering a mixed $\mathrm{BC}$ on $Q$ and the following trace condition $[57,58]$

$$
K=\frac{d}{d-1} T
$$

was obtained. The employment of a mixed BC is a reasonable assumption if one think of $Q$ as a brane and then there should be a single embedding equation for it. ${ }^{3}$ In addition, we impose a Neumann boundary condition for the gauge field,

$$
\mathcal{H}_{L M N} n_{Q}^{L} \Pi_{\alpha}^{M} \Pi_{\beta}^{N}=0 .
$$

Here $n_{Q}$ is the inward-pointing normal vector on $Q$, the beginning Greek letters $\alpha, \beta$ etc denote indices on $Q$, and $\Pi$ is the projection operator which gives the vector field and metric on $Q: \bar{B}_{\alpha \beta}=\Pi_{\alpha}^{M} \Pi_{\beta}^{N} \mathcal{B}_{M N}$ and $\gamma_{\alpha \beta}=\Pi_{\alpha}^{M} \Pi_{\beta}^{N} G_{M N}$. We note that [50] the manifold $N$ is actually singular since the normal of $N$ is discontinuous at the junction $P$. Due to this discontinuity, an expansion in small $z$ in the form of Fefferman-Graham (FG) asymptotic expansion [78] would not be sufficient, and one needs to have a full analytic control of the metric near $P$, i.e. near $z=0=x$. The need of a non-FG expanded bulk metric was already anticipated in [60]. The general form of this non-FG expanded bulk metric that is analytic near $P$ was successfully constructed in [50] by considering an expansion in small exterior curvature of the boundary surface $P$. Moreover it was found that by using the non-FG expansion of the metric in the bulk, the tensor embedding equation

$$
K_{\alpha \beta}-(K-T) \gamma_{\alpha \beta}=0
$$

for $Q$ as proposed originally by Takayanagai [56] is also consistent [50]: with the tensor model (3.6) considered as a special case of the scalar model (3.4).

Now back to our system and let us solve for the shape $Q$ and the gauge field configuration. Let us denote the 7 -dimensional bulk indices by $S=(z, \mu)$, and the 6 -dimensional field theory indices by $\mu=(x, a)$ with $a=0,1, \cdots, 4$. For simplicity, let us consider the case of a flat half space $x \geq 0$. The bulk metric reads

$$
d s^{2}=R^{2} \frac{d z^{2}+d x^{2}+\delta_{a b} d y^{a} d y^{b}}{z^{2}}
$$

\footnotetext{
${ }^{3}$ We thank Juan Maldacena for suggesting this interpretation.
} 
where $R$ is the $A d S_{7}$ radius. In this case, (3.6) reduces to (3.4), and $Q$ is given by [56]

$$
x=-z \sinh \frac{\rho}{R},
$$

where we have re-parametrized $T=\frac{5}{R} \tanh \frac{\rho}{R}$.

As for the solution for the gauge field, we will consider the situation of having a constant field strength in the BCFT. Due to the planar symmetry of the boundary, we consider $\mathcal{B}_{M N}$ that depends only on the coordinates $z$ and $x$. The field equations $\nabla_{L} \mathcal{H}^{L M N}=0$ can be solved with non-vanishing components $\mathcal{B}_{z a}=\mathcal{B}_{z a}(z), \mathcal{B}_{x a}=\mathcal{B}_{x a}(x), \mathcal{B}_{a b}=\mathcal{B}_{a b}(z, x)$, and with $\mathcal{B}_{a b}$ satisfying,

$$
z \partial_{z}^{2} \mathcal{B}_{a b}+z \partial_{x}^{2} \mathcal{B}_{a b}-\partial_{z} \mathcal{B}_{a b}=0
$$

To solve this, let us take the ansatz

$$
\mathcal{B}_{a b}=\sum_{n=0} x^{n} f_{n}\left(\frac{z}{x}\right) B_{a b}^{(n)}
$$

where $f_{n}(0)=1$ so that $\mathcal{B}_{a b}$ reduces to the gauge field $B_{a b}$ at the AdS boundary $z=0$. Here the constants $B_{a b}^{(n)}$ 's are the expansion coefficients of $B_{a b}$ about the boundary $x=0$ :

$$
B_{a b}=\sum_{n=0} x^{n} B_{a b}^{(n)} .
$$

Considering the case of a constant field strength $H_{x a b}$ in BCFT. In this case, we have a non-vanishing $B_{a b}^{(1)}$ given by

$$
B_{a b}^{(1)}=H_{x a b}
$$

and the equation of motion (3.9) has the solution $\mathcal{B}_{a b}=x f\left(\frac{z}{x}\right) H_{x a b}$ with $f(s)=\left(1-c_{1}\right)+$ $c_{1} \sqrt{1+s^{2}}$. The boundary condition (3.5) then imposes that $c_{1}=1$ and

$$
\mathcal{B}_{a b}=H_{x a b} \sqrt{x^{2}+z^{2}} .
$$

This give rises to the non-vanishing components

$$
\mathcal{H}_{z a b}=H_{x a b} \frac{z}{\sqrt{x^{2}+z^{2}}}, \quad \mathcal{H}_{x a b}=H_{x a b} \frac{x}{\sqrt{x^{2}+z^{2}}}
$$

for the bulk field strength. From the gravitational action (3.1), one then derive the holographic current

$$
\left\langle J^{a b}\right\rangle=\lim _{z \rightarrow 0} \frac{\delta I}{\delta \mathcal{B}_{a b}}=b \frac{H_{x a b}}{x},
$$

where

$$
b=-\frac{R}{16 \pi G_{N} q^{2}}
$$

is a constant. It is remarkable that the current (3.15) is independent of the parameter $T$, showing that the boundary current in $6 \mathrm{~d}$ BCFT is independent of boundary conditions. 


\section{Weyl anomaly from boundary current}

Recall that for 4-dimensional BCFT, the following relation can be established [1]

$$
(\delta \mathcal{A})_{\partial M}=\left(\int_{M_{\epsilon}} J^{\mu} \delta A_{\mu}\right)_{\log \frac{1}{\epsilon}} .
$$

Here $\epsilon>0$ is a UV regulator and $M_{\epsilon}$ is the regulated manifold with $x \geq \epsilon$. The relation (4.1) relates the boundary term of the variation of the Weyl anomaly under an arbitrary variation of the vector gauge field $\delta A_{\mu}$ with the coefficient of the logarithmic divergent term of the regulated integral on the right hand side. In general the Weyl anomaly can be computed from the quantum effects of matter loops on the path integral with external gauge fields. The relation (4.1) then allows one to determine the form of the current [1] (and also the stress tensor [50]) near the boundary. Vice versa, one may also use the current as determined by holography as input and use it to determine the Weyl anomaly. The results are of course all consistent with each other.

In higher dimensions, the gauge field contribution to the Weyl anomaly is unknown. Nevertheless, even without any knowledge of the path integral or how the higher rank gauge field, one can easily generalize the analysis of [1] and establish a similar relation (4.1) between the Weyl anomaly and the boundary current. couples to the other fields of the system. To be concrete, let us consider $d=6$. In this case, for a conserved 2 -form current $J^{\mu \nu}, \partial_{\mu} J^{\mu \nu}=0$ coupled to an external 2-form tensor gauge field $B_{\mu \nu}$ with the coupling (2.2), we have the relation

$$
(\delta \mathcal{A})_{\partial M_{\epsilon}}=\left(\int_{M_{\epsilon}} J^{\mu \nu} \delta B_{\mu \nu}\right)_{\log \frac{1}{\epsilon}} .
$$

For completeness, we give a proof of (4.2) in the appendix of the paper. Using the holographic result (3.15), and for a generic field strength, one can verify that (4.1) is satisfied with $\mathcal{A}$ given by

$$
\mathcal{A}=\int_{M} \frac{b}{6} H_{\mu \nu \lambda}^{2}
$$

We remark that one can also use the AdS/BCFT to compute the holographic stress tensor and the Weyl anomaly $[57,58]$. The same result is obtained. This is our prediction for the form of the gauge field contribution in the Weyl anomaly in 6d CFT with tensor gauge field.

An interesting application is in the theory of multiple M5-branes in M-theory. Consider a system of $N$ coincident M5-branes in flat space. Although the field theoretic description of the six-dimensional $(2,0)$ superconformal field theory is unknown, nevertheless it is possible to give a holographic description of the system by M-theory on an $A d S_{7} \times S^{4}$ background. The supergravity background is given by a constant 4 -form field strength and the metric

$$
d s^{2}=R^{2} \frac{d z^{2}+d x_{6}^{2}}{z^{2}}+R^{\prime 2} d \Omega_{4}^{2}
$$

where $R=2(\pi N)^{1 / 3} l_{11}$ is the AdS radius, $R^{\prime}=R / 2$ and $l_{11}$ is the 11-dimensional Planck length. We note that ${ }^{4}$ the spectrum of KK reduction of the eleven-dimensional supergravity

\footnotetext{
${ }^{4}$ We thank the referee for this comment.
} 
on $S^{4}$ contains a massive three-form gauge fields which obeys an "odd dimensional selfduality" condition, or equivalently, two massless two-form gauge fields under a Hodge duality [79]. Therefore, at least in the large $N$ limit, AdS/CFT predicts the existence of two global Abelian 2-form currents and corespondingly two Abelian global symmetries in the $(2,0)$ superconformal field theory. It is intriguing to note that there are indeed two $\mathrm{U}(1)$ global symmetries within the symmeries of the non-abelian tensor gauge fields in the $\mathrm{U}(N) \times \mathrm{U}(N)$ construction of [23]. Such a gauge symmetry in the $(2,0)$ theory has been predicted to arise from the $\mathrm{U}(N) \times \mathrm{U}(N)$ Kac-Moody symmetry of the multiple self-dual strings worldsheet on the M5-branes [22].

The existence of these global currents is interesting and one can exploit their properties to learn something about the $(2,0)$ theory. To do this, let us introduce a boundary in the M5-branes system. Our AdS/CFT analysis as performed in the previous section predicts a holographic boundary current $(3.15)$ in the $(2,0)$ theory in the presence of an external 3 -form flux $H$. As the supergravity is maximally supersymmetric, the constant $1 / q^{2}$ in the supergravity action is not an independent scale, but is related to the AdS radius

$$
1 / q^{2} \sim R^{4}
$$

up to a dimensionless numerical constant. Since the 7-dimensional Newton constant $G_{N}=$ $G_{N}^{(11)} / \operatorname{Vol}\left(S^{4}\right)$ and $G_{N}^{(11)}=16 \pi^{7} l_{11}^{9}$, we obtain

$$
b \sim-\left(R / l_{11}\right)^{9} \sim-N^{3}
$$

for the two Abelian 2-form currents in the $(2,0)$ theory that are dual to the two massless KK 2-form gauge fields in the 7-dimensional bulk supergravity.

We notice that in 4-dimensions, the coefficient $b$ of the boundary current is given by the beta function of the theory and it is proportional to the number of degree of freedom that couple to the U(1) gauge field. Here we expect that $b$ to be proportional to the degrees of freedom that couple to the 2-form gauge field. Our result (4.6) suggests that an order of $N^{3}$ degree of freedom couples to the external $B_{\mu \nu}$ field and the number of degree of freedom in the $(2,0)$ theory is proportional to $N^{3}$ for large $N$. We note that a scaling dependence of $N^{3}$ also appear in the entropy of a system of coincident near extremal black 5-branes solution [42]. However we emphasis that the associated physical mechanism is different: here there is no horizon in the geometry and a different observable, a conserved current, is considered.

\section{Discussion}

In this paper we have derived the existence and the form of a boundary two-form current in the presence of a background 3-form flux in a 6-dimensional CFT. The background 3-form flux also induces a Weyl anomaly in the theory. We derived these results using holographic principle. An interesting question is whether and how one may understand these results in terms of field theory directly.

In 4-dimensions, the induced boundary current aroused from the magnetization effect of the renormalized vacuum near the boundary. Both the current and the Weyl anomaly 
came from the quantum loop effects of matter fields in the presence of an external gauge field background. The current (3.15) and the Weyl-anomaly (4.3) for 6-dimensions should have a similar origin. Note that there is no obvious way to couple a point particle to a tensor gauge field $B_{\mu \nu}$ in 6-dimensions. However there is a natural way to couple $B$ to a string. To see this, let us recall that in 4-dimensions, the coupling of matter field to external gauge field $A_{\mu}$ can be obtained by gauging the global symmetry of the theory. For example, the $\mathrm{U}(1)$ gauge symmetry of a Dirac fermion field

$$
\psi(x) \rightarrow e^{i \alpha} \psi(x)
$$

gives rise to the covariant derivative $D_{\mu} \psi=\left(\partial_{\mu}-i A_{\mu}\right) \psi$ and the current $J^{\mu}=\bar{\psi} \gamma^{\mu} \psi$. In the same way there is a natural way to construct a covariant derivative for a tensor gauge potential if it is represented on a functional $\Psi(C)$ of string/loop. First we define the loop derivative

$$
\partial_{\mu \nu} \Psi:=\frac{\partial \Psi(C)}{\partial \sigma^{\mu \nu}}:=\lim _{\delta \sigma^{\mu \nu} \rightarrow 0} \frac{\Psi(C+\delta C)-\Psi(C)}{\delta \sigma^{\mu \nu}},
$$

where $\delta \sigma^{\mu \nu}$ is the infinitesimal area element caused by the infinitesimal change in the loop. The derivative exists whenever the limit is well defined. $\partial_{\mu \nu}$ is antisymmetric in the indices $\mu, \nu$. In general, an arbitrary change in the phase of the string functional takes the form

$$
\Psi(C) \rightarrow \Psi(C) \exp \left(i \int_{C} \alpha\right)
$$

where $\alpha=\alpha_{\mu} d x^{\mu}$ is an arbitrary 1-form. It is easy to check that the derivative defined by

$$
\mathcal{D}_{\mu \nu} \Psi:=\left(\partial_{\mu \nu}-i B_{\mu \nu}\right) \Psi
$$

transforms covariantly if $B_{\mu \nu}$ transforms as

$$
B_{\mu \nu} \rightarrow B_{\mu \nu}+\partial_{\mu} \alpha_{\nu}-\partial_{\nu} \alpha_{\mu}
$$

Using the covariant derivative (5.4), one may consider the string field action

$$
S=\int[D x(\sigma)]\left(D_{\mu \nu} \Phi D^{\mu \nu} \Phi+i \bar{\Psi} \gamma^{\mu \nu} D_{\mu \nu} \Psi\right)
$$

where $\Phi$ is a real string field, $\Psi$ is a Weyl spinor string field and the integration is over all possible closed loops. The action processes a global U(1) symmetry which gives the Noether current $J_{\mu \nu}=\bar{\Psi} \gamma_{\mu \nu} \Psi$. Our prediction is that the Weyl anomaly (4.3) would arise from the effective action of the string field theory coupled to an external 3-form flux background. We leave this problem to future work.

Another interesting question come from the following observation. Recall that Dbranes in the presence of a constant 2-form NS-NS $B$-field background is described by a non-commutative geometry of Moyal type. This can be derived by considering open string quantisation. One may expect a link between the quantum geometry on the D-brane with the magnetic Casimir effect as both consequence of the background flux . For a M5-brane in the presence of a constant 3 -form $C$-field background, it has been widely speculated that it 


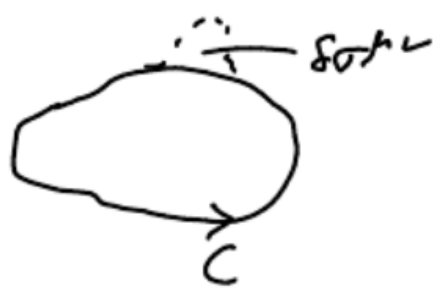

Figure 2. Loop deformation.

must give rises to some kind of non-commutative geometry in certain limit. However much of this speculation is unknown so far. All we know is that it must reduce to a Moyal type non-commutative geometry upon a dimensional reduction. It may be possible to establish an analogous relation between the desired quantum geometry and the boundary 2-form current and use the magnetic Casimir effect to learn about the quantum geometry.

\section{Acknowledgments}

CSC would like to thank David Berman, Jack Distler, Neil Lambert, Yutaka Masuo and Douglas Smith for useful comments and questions, and for the Institute of Mathemaical Sciences, Singapore for hospitality during the workshop "String and M-Theory: The New Geometry of the 21st Century", where part of this work was carried out. This work has been supported in part by the National Center of Theoretical Science (NCTS) and the grant 107-2119-M-007-014-MY3 of the Ministry of Science and Technology of Taiwan.

\section{A Derivation of key relation (4.2)}

Consider a BQFT with a well defined effective action. The integrated Weyl anomaly $\mathcal{A}$ [80]

$$
\mathcal{A}=\int_{M} \sqrt{g}\left[g^{\mu \nu}\left\langle T_{\mu \nu}\right\rangle-\left\langle g^{\mu \nu} T_{\mu \nu}\right\rangle\right]
$$

can be obtained as the coefficient of the logarithmic UV divergent term of the expectation value of the effective action,

$$
I=\cdots+\mathcal{A} \log \left(\frac{1}{\epsilon}\right)+I_{\text {finite }}
$$

where $\cdots$ denotes terms which are UV divergent in powers of the UV cutoff $1 / \epsilon$, and $I_{\text {finite }}$ is the renormalized, UV finite part of the effective action. To derive this result, let us consider a constant Weyl transformation $g_{\mu \nu} \rightarrow \exp (2 \omega) g_{\mu \nu}$. Under this transformation, the UV cutoff transforms as $\epsilon \rightarrow \exp (\omega) \epsilon$ and the variation of effective action (A.2) becomes

$$
\delta_{\omega} I=\cdots+\omega\left(-\mathcal{A}+\int_{M} \sqrt{g}\left\langle T^{\mu \nu}\right\rangle g_{\mu \nu}\right)+O\left(\omega^{2}\right)
$$


where we have used $\delta_{\omega} \mathcal{A}=0$ and $\delta_{\omega} I_{\text {finite }}=\omega \int_{M} \sqrt{g}\left\langle T^{\mu \nu}\right\rangle g_{\mu \nu}+O\left(\omega^{2}\right)$. On the other hand, by definition we have

$$
\delta_{\omega} I=\frac{1}{2} \int_{M} \sqrt{g} \hat{T}^{\mu \nu} \delta_{\omega} g_{\mu \nu}=\omega \int_{M} \sqrt{g} \hat{T}^{\mu \nu} g_{\mu \nu}+O\left(\omega^{2}\right)
$$

where $\hat{T}^{\mu \nu}$ is the non-renormalized stress tensor. We use the hatted symbol (e.g. $\hat{T}_{\mu \nu}$ ) to denote non-renormalized quantity and un-hatted symbol (e.g. $T_{\mu \nu}$ ) to denote renormalized quantity. Separating $\hat{T}^{\mu \nu} g_{\mu \nu}$ into the renormalized UV finite part $\left\langle\hat{T}^{\mu \nu} g_{\mu \nu}\right\rangle$ and divergent part, we have

$$
\delta_{\omega} I=\cdots+\omega \int_{M} \sqrt{g}\left\langle\hat{T}^{\mu \nu} g_{\mu \nu}\right\rangle+O\left(\omega^{2}\right)
$$

Comparing the finite part of (A.3) and (A.5), we obtain the expression (A.1) for $\mathcal{A}$ and hence our claim.

Now we are ready to prove the result (4.1) quoted in the main text of the paper. As in [1], let us regulate the effective action by excluding from its volume integration a small strip of geodesic distance $\epsilon$ from the boundary. Then there is no explicit boundary divergences in this form of the effective action, however there are boundary divergences implicit in the bulk effective action which is integrated up to distance $\epsilon$. The variation of effective action with respect to the 2 -form potential is given by

$$
\delta I=\int_{x \geq \epsilon} \sqrt{g} \hat{J}^{\mu \nu} \delta B_{\mu \nu}
$$

where $\hat{J}^{\mu \nu}=\frac{\delta I}{\sqrt{g} \delta B_{\mu \nu}}$ is the non-renormalized bulk current. The renormalized bulk current is defined by the difference of the non-renormalized bulk current against a reference one [81]:

$$
J^{\mu \nu}=\hat{J}^{\mu \nu}-\hat{J}_{0}^{\mu \nu},
$$

where $\hat{J}_{0}^{\mu \nu}$ is the non-renormalized current defined for the same CFT without boundary. It is

$$
\delta I_{0}=\int_{x \geq \epsilon} \sqrt{g} \hat{J}_{0}^{\mu \nu} \delta B_{\mu \nu}
$$

where $I_{0}$ is the effective action of the CFT with the boundary removed, hence the integration over the region $x \geq \epsilon$. Subtract (A.8) from (A.6) and focus on only the logarithmically divergent terms, we obtain our key formula

$$
(\delta \mathcal{A})_{\partial M}=\left(\int_{x \geq \epsilon} \sqrt{g} J^{\mu \nu} \delta B_{\mu \nu}\right)_{\log (1 / \epsilon)}
$$

where $(\delta \mathcal{A})_{\partial M}$ is the boundary terms in the variations of Weyl anomaly and $J^{\mu}$ is the renormalized bulk current. In the above derivations, we have used the fact that $\delta I$ and $\delta I_{0}$ have the same bulk variation of Weyl anomaly so that

$$
(\delta \mathcal{A})_{\partial M}=\left(\delta I-\delta I_{0}\right)_{\log (1 / \epsilon)} .
$$




\section{B Holographic Weyl anomaly}

In this appendix, we investigate the holographic Weyl anomaly for 6d CFT. Since we are interested in the bulk Weyl anomaly (4.3) which is irrelevant to the boundary, we focus on the case without boundary. For simplicity, we focus on the flat space. Then all the curvatures vanish and only the field strength $H_{i j k}$ contribute to Weyl anomaly. According to [82], holographic Weyl anomaly can be obtained as the UV logarithmic divergent terms of the gravitational action (3.1). In the FG gauge, we have

$$
d s^{2}=G_{M N} d x^{M} d x^{N}=\frac{d z^{2}+\hat{g}_{\mu \nu} d x^{\mu} d x^{\nu}}{z^{2}},
$$

where $\hat{g}_{\mu \nu}=g_{\mu \nu}+z^{2} g_{\mu \nu}^{(2)}+z^{4} g_{\mu \nu}^{(4)}+\cdots$. Since we focus on flat space $g_{\mu \nu}=\eta_{\mu \nu}$, we have $g_{\mu \nu}^{(2)}=0[82,83]$. According to [84], $g_{\mu \nu}^{(4)}$ and higher order terms in the expansions of $g_{\mu \nu}$ do not contribute to holographic Weyl anomaly for $6 \mathrm{~d}$ CFT. Thus, we can set $\hat{g}_{\mu \nu}=\eta_{\mu \nu}$ in the following derivations. Similar to FG gauge (B.1) for the bulk metric, we take the following gauge for bulk gauge field

$$
\mathcal{B}_{z \mu}=0, \quad \mathcal{B}_{\mu \nu}=B_{\mu \nu}+z^{2} B_{\mu \nu}^{(2)}+\cdots
$$

where $B_{\mu \nu}$ is the background gauge field for $6 \mathrm{~d}$ CFT.

Substituting (B.1), (B.2), together with $\hat{g}_{\mu \nu}=\eta_{\mu \nu}$ into the action (3.3), we obtain the logarithmic divergent term as

$$
\begin{aligned}
I & =\frac{1}{16 \pi G_{N}} \int d z d^{6} x \frac{\sqrt{g}}{z^{7}}\left(\cdots-\frac{z^{6}}{6} H_{\mu \nu \lambda} H_{\alpha \beta \rho} g^{\mu \alpha} g^{\nu \beta} g^{\lambda \rho}\right), \\
& =-\frac{1}{96 \pi G_{N}} \int_{M} d^{6} x \sqrt{g} H_{\mu \nu \lambda}^{2} \ln \frac{1}{\epsilon}+\cdots
\end{aligned}
$$

where $\cdots$ denote power law divergent terms and regular terms. From (B.3), we can read off the holographic Weyl anomaly

$$
\mathcal{A}=\int_{M} \sqrt{g} \frac{b}{6} H_{\mu \nu \lambda}^{2}
$$

with $b=-\frac{R}{16 \pi G_{N} q^{2}}$. This holographic Weyl anomaly agrees precisely with that obtained in (4.3).

Open Access. This article is distributed under the terms of the Creative Commons Attribution License (CC-BY 4.0), which permits any use, distribution and reproduction in any medium, provided the original author(s) and source are credited.

\section{References}

[1] C.-S. Chu and R.-X. Miao, Weyl anomaly induced current in boundary quantum field theories, Phys. Rev. Lett. 121 (2018) 251602 [arXiv:1803.03068] [INSPIRE].

[2] C.-S. Chu and R.-X. Miao, Anomalous transport in holographic boundary conformal field theories, JHEP 07 (2018) 005 [arXiv:1804.01648] [INSPIRE]. 
[3] E. Witten, Some comments on string dynamics, hep-th/9507121 [INSPIRE].

[4] P.S. Howe and E. Sezgin, $D=11, p=5$, Phys. Lett. B 394 (1997) 62 [hep-th/9611008] [INSPIRE].

[5] P.S. Howe, E. Sezgin and P.C. West, Covariant field equations of the M-theory five-brane, Phys. Lett. B 399 (1997) 49 [hep-th/9702008] [INSPIRE].

[6] M. Perry and J.H. Schwarz, Interacting chiral gauge fields in six-dimensions and Born-Infeld theory, Nucl. Phys. B 489 (1997) 47 [hep-th/9611065] [INSPIRE].

[7] M. Aganagic, J. Park, C. Popescu and J.H. Schwarz, World volume action of the M-theory five-brane, Nucl. Phys. B 496 (1997) 191 [hep-th/9701166] [INSPIRE].

[8] I.A. Bandos et al., Covariant action for the superfive-brane of M-theory, Phys. Rev. Lett. 78 (1997) 4332 [hep-th/9701149] [INSPIRE].

[9] I.A. Bandos et al., On the equivalence of different formulations of the M-theory five-brane, Phys. Lett. B 408 (1997) 135 [hep-th/9703127] [INSPIRE].

[10] C.-S. Chu and S.-L. Ko, Non-abelian action for multiple five-branes with self-dual tensors, JHEP 05 (2012) 028 [arXiv: 1203.4224] [INSPIRE].

[11] C.-S. Chu, S.-L. Ko and P. Vanichchapongjaroen, Non-abelian self-dual string solutions, JHEP 09 (2012) 018 [arXiv: 1207.1095] [INSPIRE].

[12] C.-S. Chu and P. Vanichchapongjaroen, Non-abelian self-dual string and M2-M5 branes intersection in supergravity, JHEP 06 (2013) 028 [arXiv: 1304.4322] [INSPIRE].

[13] C.-S. Chu and H. Isono, Instanton wave and M-wave in multiple M5-branes system, Eur. Phys. J. C 73 (2013) 2586 [arXiv: 1305.6808] [INSPIRE].

[14] V. Niarchos and K. Siampos, M2-M5 blackfold funnels, JHEP 06 (2012) 175 [arXiv: 1205.1535] [INSPIRE].

[15] V. Niarchos and K. Siampos, The black M2-M5 ring intersection spins, PoS( Corfu2012) 088 [arXiv:1302.0854] [INSPIRE].

[16] O. Aharony et al., Matrix description of interacting theories in six-dimensions, Adv. Theor. Math. Phys. 1 (1998) 148 [hep-th/9707079] [INSPIRE].

[17] O. Aharony, M. Berkooz and N. Seiberg, Light cone description of $(2,0)$ superconformal theories in six-dimensions, Adv. Theor. Math. Phys. 2 (1998) 119 [hep-th/9712117] [INSPIRE].

[18] N. Arkani-Hamed, A.G. Cohen, D.B. Kaplan, A. Karch and L. Motl, Deconstructing $(2,0)$ and little string theories, JHEP 01 (2003) 083 [hep-th/0110146] [INSPIRE].

[19] M.R. Douglas, On D = 5 super Yang-Mills theory and (2,0) theory, JHEP 02 (2011) 011 [arXiv: 1012.2880] [INSPIRE].

[20] N. Lambert, C. Papageorgakis and M. Schmidt-Sommerfeld, M5-branes, D4-branes and quantum 5D super-Yang-Mills, JHEP 01 (2011) 083 [arXiv:1012.2882] [INSPIRE].

[21] C.-S. Chu and D.J. Smith, Towards the quantum geometry of the M5-brane in a constant $C$-field from multiple membranes, JHEP 04 (2009) 097 [arXiv:0901.1847] [INSPIRE].

[22] C.-S. Chu and D.J. Smith, Multiple self-dual strings on M5-branes, JHEP 01 (2010) 001 [arXiv: 0909.2333] [INSPIRE].

[23] C.-S. Chu, A theory of non-Abelian tensor gauge field with non-Abelian gauge symmetry $G \times G$, Nucl. Phys. B 866 (2013) 43 [arXiv:1108.5131] [InSPIRE]. 
[24] P.M. Ho and Y. Matsuo, Aspects of effective theory for multiple M5-branes compactified on circle, JHEP 12 (2014) 154 [arXiv:1409.4060] [INSPIRE].

[25] P.-M. Ho, K.-W. Huang and Y. Matsuo, A non-Abelian self-dual gauge theory in $5+1$ dimensions, JHEP 07 (2011) 021 [arXiv:1104.4040] [INSPIRE].

[26] H. Samtleben, E. Sezgin and R. Wimmer, $(1,0)$ superconformal models in six dimensions, JHEP 12 (2011) 062 [arXiv:1108.4060] [INSPIRE].

[27] H.-C. Kim, S. Kim, E. Koh, K. Lee and S. Lee, On instantons as Kaluza-Klein modes of M5-branes, JHEP 12 (2011) 031 [arXiv:1110.2175] [INSPIRE].

[28] J. Kallen, J.A. Minahan, A. Nedelin and M. Zabzine, $N^{3}$-behavior from 5D Yang-Mills theory, JHEP 10 (2012) 184 [arXiv:1207.3763] [INSPIRE].

[29] Z. Bern et al., D =5 maximally supersymmetric Yang-Mills theory diverges at six loops, Phys. Rev. D 87 (2013) 025018 [arXiv:1210.7709] [INSPIRE].

[30] C. Cordova, T.T. Dumitrescu and X. Yin, Higher derivative terms, toroidal compactification and Weyl anomalies in six-dimensional $(2,0)$ theories, arXiv: 1505.03850 [INSPIRE].

[31] C. Beem, M. Lemos, L. Rastelli and B.C. van Rees, The $(2,0)$ superconformal bootstrap, Phys. Rev. D 93 (2016) 025016 [arXiv: 1507.05637] [InSPIRE].

[32] K. Hosomichi, R.-K. Seong and S. Terashima, Supersymmetric gauge theories on the five-sphere, Nucl. Phys. B 865 (2012) 376 [arXiv:1203.0371] [InSPIRE].

[33] J. Källén, J. Qiu and M. Zabzine, The perturbative partition function of supersymmetric $5 D$ Yang-Mills theory with matter on the five-sphere, JHEP 08 (2012) 157 [arXiv:1206.6008] [INSPIRE].

[34] H.-C. Kim and S. Kim, M5-branes from gauge theories on the 5-sphere, JHEP 05 (2013) 144 [arXiv: 1206.6339] [INSPIRE].

[35] H.-C. Kim, S.-S. Kim and K. Lee, 5-dim superconformal index with enhanced En global symmetry, JHEP 10 (2012) 142 [arXiv:1206.6781] [INSPIRE].

[36] H.-C. Kim, J. Kim and S. Kim, Instantons on the 5-sphere and M5-branes, arXiv: 1211.0144 [INSPIRE].

[37] C. Sämann and M. Wolf, Non-abelian tensor multiplet equations from twistor space, Commun. Math. Phys. 328 (2014) 527 [arXiv:1205.3108] [InSPIRE].

[38] C. Sämann, Lectures on higher structures in M-theory, arXiv:1609.09815 [INSPIRE].

[39] A. Strominger, Open p-branes, Phys. Lett. B 383 (1996) 44 [hep-th/9512059] [INSPIRE].

[40] R. Dijkgraaf, E.P. Verlinde and H.L. Verlinde, BPS quantization of the five-brane, Nucl. Phys. B 486 (1997) 89 [hep-th/9604055] [INSPIRE].

[41] Y. Tachikawa, On S-duality of 5d super Yang-Mills on $S^{1}$, JHEP 11 (2011) 123 [arXiv:1110.0531] [INSPIRE].

[42] I.R. Klebanov and A.A. Tseytlin, Entropy of near extremal black p-branes, Nucl. Phys. B 475 (1996) 164 [hep-th/9604089] [INSPIRE].

[43] C.-S. Chu and P.-M. Ho, Noncommutative open string and D-brane, Nucl. Phys. B 550 (1999) 151 [hep-th/9812219] [INSPIRE].

[44] C.-S. Chu and P.-M. Ho, Constrained quantization of open string in background B field and noncommutative D-brane, Nucl. Phys. B 568 (2000) 447 [hep-th/9906192] [INSPIRE].

[45] J.L. Cardy, Boundary conformal field theory, hep-th/0411189 [InSPIRE]. 
[46] M.Z. Hasan and C.L. Kane, Topological Insulators, Rev. Mod. Phys. 82 (2010) 3045 [arXiv: 1002.3895] [INSPIRE].

[47] D.M. McAvity and H. Osborn, Energy momentum tensor in conformal field theories near a boundary, Nucl. Phys. B 406 (1993) 655 [hep-th/9302068] [INSPIRE].

[48] D. Fursaev, Conformal anomalies of CFT's with boundaries, JHEP 12 (2015) 112 [arXiv: 1510.01427] [INSPIRE].

[49] C.P. Herzog, K.-W. Huang and K. Jensen, Universal entanglement and boundary geometry in conformal field theory, JHEP 01 (2016) 162 [arXiv:1510.00021] [INSPIRE].

[50] R.-X. Miao and C.-S. Chu, Universality for shape dependence of Casimir effects from Weyl anomaly, JHEP 03 (2018) 046 [arXiv:1706.09652] [INSPIRE].

[51] C. Herzog, K.-W. Huang and K. Jensen, Displacement operators and constraints on boundary central charges, Phys. Rev. Lett. 120 (2018) 021601 [arXiv:1709.07431] [INSPIRE].

[52] K. Jensen, E. Shaverin and A. Yarom, 't Hooft anomalies and boundaries, JHEP 01 (2018) 085 [arXiv: 1710.07299] [inSPIRE].

[53] M. Kurkov and D. Vassilevich, Parity anomaly in four dimensions, Phys. Rev. D 96 (2017) 025011 [arXiv: 1704.06736] [INSPIRE].

[54] M. Kurkov and D. Vassilevich, Gravitational parity anomaly with and without boundaries, JHEP 03 (2018) 072 [arXiv: 1801.02049] [INSPIRE].

[55] D. Rodriguez-Gomez and J.G. Russo, Free energy and boundary anomalies on $\mathbb{S}^{a} \times \mathbb{H}^{b}$ spaces, JHEP 10 (2017) 084 [arXiv: 1708.00305] [INSPIRE].

[56] T. Takayanagi, Holographic dual of BCFT, Phys. Rev. Lett. 107 (2011) 101602 [arXiv:1105.5165] [INSPIRE].

[57] R.-X. Miao, C.-S. Chu and W.-Z. Guo, New proposal for a holographic boundary conformal field theory, Phys. Rev. D 96 (2017) 046005 [arXiv:1701.04275] [INSPIRE].

[58] C.-S. Chu, R.-X. Miao and W.-Z. Guo, On new proposal for holographic BCFT, JHEP 04 (2017) 089 [arXiv: 1701.07202] [INSPIRE].

[59] M. Fujita, T. Takayanagi and E. Tonni, Aspects of AdS/BCFT, JHEP 11 (2011) 043 [arXiv:1108.5152] [INSPIRE].

[60] M. Nozaki, T. Takayanagi and T. Ugajin, Central charges for BCFTs and holography, JHEP 06 (2012) 066 [arXiv: 1205.1573] [INSPIRE].

[61] A. Faraji Astaneh and S.N. Solodukhin, Holographic calculation of boundary terms in conformal anomaly, Phys. Lett. B 769 (2017) 25 [arXiv:1702.00566] [INSPIRE].

[62] M. Flory, A complexity/fidelity susceptibility g-theorem for $A d S_{3} / B C F T_{2}, J H E P 06$ (2017) 131 [arXiv: 1702.06386] [INSPIRE].

[63] S. Bhowmick, S. Das and B. Ezhuthachan, Entanglement entropy and kinematic space in $B C F T$ and $R G$ flow, arXiv:1703.01759 [INSPIRE].

[64] C.P. Herzog and K.-W. Huang, Boundary conformal field theory and a boundary central charge, JHEP 10 (2017) 189 [arXiv:1707.06224] [INSPIRE].

[65] D. Seminara, J. Sisti and E. Tonni, Corner contributions to holographic entanglement entropy in $A d S_{4} / B C F T_{3}$, JHEP 11 (2017) 076 [arXiv: 1708.05080] [INSPIRE].

[66] E.-J. Chang, C.-J. Chou and Y. Yang, Holographic entanglement entropy in boundary conformal field theory, Phys. Rev. D 98 (2018) 106016 [arXiv:1805.06117] [INSPIRE]. 
[67] D. Seminara, J. Sisti and E. Tonni, Holographic entanglement entropy in $A d S_{4} / B C F T_{3}$ and the Willmore functional, JHEP 08 (2018) 164 [arXiv:1805.11551] [INSPIRE].

[68] R.-X. Miao, Holographic BCFT with Dirichlet boundary condition, JHEP 02 (2019) 025 [arXiv: 1806.10777] [INSPIRE].

[69] N. Andrei et al., Boundary and defect CFT: open problems and applications, arXiv: 1810.05697 [INSPIRE].

[70] H.B.G. Casimir, On the Attraction Between Two Perfectly Conducting Plates, Indag. Math. 10 (1948) 261 [Kon. Ned. Akad. Wetensch. Proc. 51 (1948) 793] [Front. Phys. 65 (1987) 342] [Kon. Ned. Akad. Wetensch. Proc. 100N3-4 (1997) 61] [InSPIRE].

[71] G. Plunien, B. Müller and W. Greiner, The Casimir effect, Phys. Rept. 134 (1986) 87 [INSPIRE].

[72] M. Bordag, U. Mohideen and V.M. Mostepanenko, New developments in the Casimir effect, Phys. Rept. 353 (2001) 1 [quant-ph/0106045] [INSPIRE].

[73] S. Deser and A. Schwimmer, Geometric classification of conformal anomalies in arbitrary dimensions, Phys. Lett. B 309 (1993) 279 [hep-th/9302047] [INSPIRE].

[74] M.E. Peskin and D.V. Schroeder, An introduction to quantum field theory, Westview Press, U.S.A. (1993).

[75] O.J. Ganor and S. Sethi, New perspectives on Yang-Mills theories with sixteen supersymmetries, JHEP 01 (1998) 007 [hep-th/9712071] [INSPIRE].

[76] T. Maxfield and S. Sethi, The conformal anomaly of M5-branes, JHEP 06 (2012) 075 [arXiv: 1204.2002] [INSPIRE].

[77] C. Beem, L. Rastelli and B.C. van Rees, $\mathcal{W}$ symmetry in six dimensions, JHEP 05 (2015) 017 [arXiv: 1404.1079] [INSPIRE].

[78] C. Fefferman and C. Robin Graham, Conformal invariants, in Elie Cartan et les Mathematiques daujourdhui, Asterisque (1985) 95.

[79] H. Samtleben and M. Weidner, The maximal D $=7$ supergravities, Nucl. Phys. B 725 (2005) 383 [hep-th/0506237] [INSPIRE].

[80] M.J. Duff, Twenty years of the Weyl anomaly, Class. Quant. Grav. 11 (1994) 1387 [hep-th/9308075] [INSPIRE].

[81] D. Deutsch and P. Candelas, Boundary effects in quantum field theory, Phys. Rev. D 20 (1979) 3063 [INSPIRE].

[82] M. Henningson and K. Skenderis, The holographic Weyl anomaly, JHEP 07 (1998) 023 [hep-th/9806087] [INSPIRE].

[83] C. Imbimbo, A. Schwimmer, S. Theisen and S. Yankielowicz, Diffeomorphisms and holographic anomalies, Class. Quant. Grav. 17 (2000) 1129 [hep-th/9910267] [INSPIRE].

[84] R.-X. Miao, A note on holographic Weyl anomaly and entanglement entropy, Class. Quant. Grav. 31 (2014) 065009 [arXiv: 1309.0211] [INSPIRE]. 